\title{
Reservoir-induced decoherence of resonantly excited confined polaritons
}

\author{
C. Ouellet-Plamondon, * G. Sallen, F. Morier-Genoud, D. Y. Oberli, M. T. Portella-Oberli, and B. Deveaud \\ Institute of Physics, École Polytechnique Fédérale de Lausanne (EPFL), CH-1015 Lausanne, Switzerland
}

(Received 10 October 2016; revised manuscript received 13 January 2017; published 7 February 2017)

\begin{abstract}
We report on the effect of decoherence on polariton bistability. The polariton hysteresis loop is shown to collapse in a similar way when increasing the temperature or under nonresonant excitation power. The hysteresis upward threshold is pulled to lower excitation power, whereas the downward threshold remains almost constant. This effect is explained by the population of an incoherent reservoir that induces dephasing and repulsive interaction that saturates at large densities. All experimental findings are accurately simulated with the excitonic Bloch equations and indicate that reservoir-induced dephasing can be dominant over the reservoir-induced energy blueshift.
\end{abstract}

DOI: 10.1103/PhysRevB.95.085302

\section{INTRODUCTION}

Microcavity polaritons, quasiparticles originating from strongly coupled quantum well excitons and microcavity photons, is an extremely versatile system where coherence and interaction properties are inherited from their light and matter components, respectively. They are easily controlled optically and allow, through their nonlinear optical response, one to study many-body interactions, topological defects, and other properties of quantum fluids [1].

In recent years, there has been an increasing number of proposals to use microcavity polaritons as a source of single photons [2,3], entangled photons [4-8], and as quantum computing devices [9-12]. On the one hand, the interest for using polaritons lies in their peculiar dispersion that weakens phonon scattering [13-16] or their ability to overcome dephasing by increasing the number of particles in the condensate [9,11]. On the other hand, early attempts to generate entangled photons through parametric scattering [17-19] indicate that dephasing does limit the correlations. Furthermore, a recent theory [20] predicts that, for resonantly pumped polariton condensates, phonon absorption can generate a significant exciton reservoir even at low temperature. Efficient polariton relaxation between confined modes was also observed [21,22]. Inevitably, the viability of these proposals depends on how robust polaritons are against any source of dephasing from the environment.

Although the effect of reservoir repulsive interactions on polaritons has been clearly demonstrated [23-26], the reservoir-induced dephasing (RID) has usually been neglected. Earlier studies have demonstrated that reservoir density fluctuations affect the energy and linewidth of nonresonantly pumped polariton condensates [27,28]. Recently, it was shown that dephasing may be increased under pulsed resonant excitation through the relaxation of upper polaritons that populate a reservoir [29,30]. The effect of an incoherent reservoir and the biexciton formation was also suggested to explain the apparent repulsive interaction between polaritons of opposite spins [20,31,32] evidenced in polariton multistability [33].

Concurrently, previous works have shown that zerodimensional (OD) confined polaritons are ideal systems to investigate the polariton bistability [33-36] and particularly

\footnotetext{
*clauderic.ouellet-plamondon@epfl.ch
}

its sensitivity to noise [36-38]. The former study showed that a symmetrical collapse of the polariton hysteresis loop occurs for increasing noise strength on the polariton population. Therefore, it may be expected that polariton dephasing is also reflected in the variations of the bistability thresholds, meaning we could use the polariton hysteresis loop as a tool to study polariton dephasing.

In this study, we demonstrate the sensitivity of polariton bistability to temperature. The polariton hysteresis loop collapses for temperatures of the order of the Rabi coupling, which is linked to an increasing incoherent reservoir population. These results suggest that the reservoir is populated even for $\mathrm{cw}$ resonant excitation on the lowest polariton mode, below the reservoir energy. This is confirmed by observing the same effect when purposely injecting nonresonantly excited carriers in the system at $4 \mathrm{~K}$. Simulating our experiment using the excitonic Bloch equations (EBE) coupled to a reservoir allows us to demonstrate that the collapse of the hysteresis loop is a consequence of both repulsive interactions and dephasing induced on the driven confined polaritons by excitons in the reservoir.

\section{EXPERIMENT}

The sample studied consists of a GaAs/AlAs distributed Bragg reflector (DBR) $\lambda$ microcavity with a single $\mathrm{In}_{0.04} \mathrm{Ga}_{0.96}$ As quantum well $(\mathrm{QW})$. The confined polariton modes are obtained by chemically etching the cavity spacer to create circular mesas of $6 \mathrm{~nm}$ in height, causing an in-plane confinement of the electromagnetic field [39]. We focus our attention on a $3-\mu \mathrm{m}$ mesa having a slightly positive cavity detuning of $\delta=0.2 \mathrm{meV}$ and a Rabi splitting of $\Omega_{0}=2 \Omega=$ $3.3 \mathrm{meV}$ [measured at $4.3 \mathrm{~K}$, see Figs. 1(a) and 1(b)]. The polariton ground state is excited resonantly with a circularly polarized single-mode cw Ti:sapphire laser, and the sample is maintained at low temperature in a helium flow cryostat.

A polariton bistability is obtained while exciting resonantly a single polariton mode with a blue detuned laser frequency and measuring the transmitted intensity as its input power is cycled. When the power is increased, the polariton mode blueshifts due to repulsive polariton-polariton interactions. A strong nonlinear jump of the transmitted intensity occurs when the energy shift equals the laser detuning [33,40]. As the power is decreased, the polariton mode stays locked to 

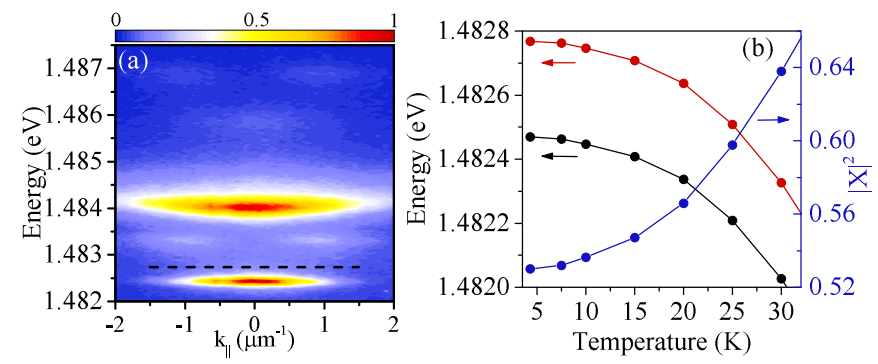

FIG. 1. (a) Photoluminescence of the 3- $\mu \mathrm{m}$ mesa as a function of in-plane wave vector and energy, measured at $4.3 \mathrm{~K}$. The black dashed line indicates the resonant laser energy. (b) Polariton groundstate energy (black) and corresponding excitonic fraction (blue) as a function of temperature. The resonant laser energy, shown in red, is kept at $\Delta=300 \mu \mathrm{eV}$ above the polariton energy.

the laser until the gain from the input laser can no longer compensate the polariton losses. The upward threshold power $P_{\text {up }}$ is therefore sensitive to the polariton density while the downward threshold power $P_{\text {down }}$ is sensitive to the polariton linewidth and dephasing. Both interaction strength and laser detuning act as scaling parameters on the system, affecting the two threshold powers.

First, we perform bistability measurements as a function of temperature. Since the polariton mode energy redshifts with increasing temperature, the laser energy is adjusted at each step to maintain a constant laser detuning $\Delta$. This fixes the blueshift needed to reach the nonlinear threshold and increases the cavity detuning and excitonic fraction [see Fig. 1(b)]; hence it increases the polariton-polariton interactions. Figure 2(a) presents the recorded hysteresis curves obtained for a laser detuning of $\Delta=300 \mu \mathrm{eV}$. We observe a drastic collapse of the bistability, the hysteresis loop being completely quenched at only $22.5 \mathrm{~K}$. This behavior is different from the effect of intensity noise for which a symmetrical collapse of the thresholds was found [36]. The threshold power for the upward and downward intensity jumps are plotted as a function of temperature in Fig. 2(b), as well as the transmitted intensity just above the upward threshold $I_{\text {up }}$. The upward threshold power $P_{\text {up }}$ decreases significantly when increasing temperature while the $P_{\text {down }}$ is barely affected. The transmitted intensity also decreases steadily with increasing temperature.

These results are unexpected because, at least for a twodimensional (2D) microcavity, polariton-phonon scattering strength from polaritons at $k=0$ is supposedly small and almost independent of temperature for temperatures below the LO-phonon energy ( 40K) [13-16]. Therefore we might expect that the effect of temperature on the bistability is minor. However, these results suggest that polariton-phonon scattering will transfer polaritons from $k=0$ to exciton states at large $k$. Then we can assume that the observed phenomenon is linked to the creation of an incoherent population of excitons in a reservoir that interacts with the resonantly excited polariton population, as suggested in Ref. [20]. Although this work studied the full 2D case, we can expect a similar phonon absorption process to occur between a confined polariton mode and a delocalized one (the exciton reservoir). It was shown theoretically in Ref. [21] that a phonon absorption process involving two OD confined polariton modes is more efficient
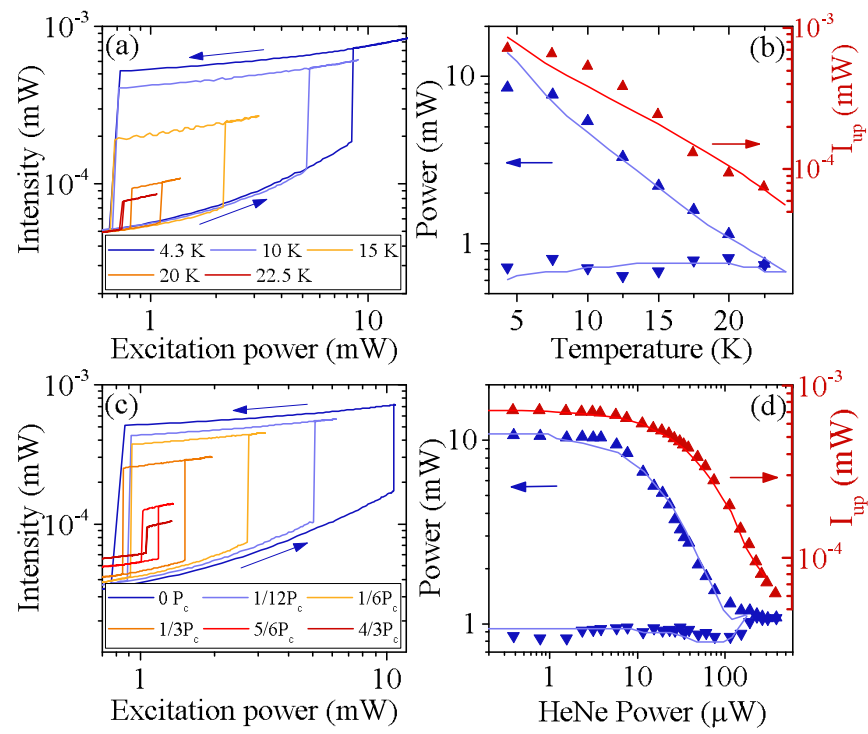

FIG. 2. Polariton bistability for $\Delta=300 \mu \mathrm{eV}$. (a) Hysteresis curves for increasing temperature. The blue arrows indicate the path when cycling the input power. (b) Excitation power at the upward and downward thresholds (blue) and transmitted intensity above the upward threshold $I_{\text {up }}$ (red) for a given temperature. The up (down) triangles correspond to the upward (downward) thresholds. The solid lines are fits using Eqs. (1) where the exciton and photon energy as well as the Rabi coupling were extracted from Fig. 1. Panels (c) and (d) is the same as in (a) and (b) but for increasing nonresonant laser power. $P_{c}=233 \mu \mathrm{W}$ represents the nonresonant power needed for the hysteresis loop to collapse.

than its $2 \mathrm{D}$ counterpart since a larger number of phonon modes contribute to the scattering. The phonon scattering strength from a delocalized exciton in the reservoir to a confined polariton mode was estimated for a $3 \mu \mathrm{m}$ mesa to be $10 \%$ of the full OD case. This suggests that the efficiency of the polaritonphonon scattering mechanism transferring a confined polariton mode to the exciton reservoir is intermediate between that of the full 2D and OD cases.

To demonstrate the effect of such a reservoir population, we repeat the experiment at a fixed temperature of $4.3 \mathrm{~K}$ while injecting an incoherent population in the reservoir in a controlled way. This is achieved experimentally by focusing a HeNe laser $(\lambda=633 \mathrm{~nm})$ onto the top surface of the mesa while the resonant laser excites the same mesa from the back of the sample. A single microscope objective is used to inject nonresonantly and to collect the transmitted resonant laser beam.

The bistability curves measured in this experimental configuration for specific HeNe powers are shown in Fig. 2(c) and the corresponding threshold power and transmitted intensity in Fig. 2(d). We observe again a rapid decrease of $P_{\text {up }}$ while $P_{\text {down }}$ is almost unaffected. Interestingly, at higher HeNe power, the decrease of $P_{\text {up }}$ levels off asymptotically. For a HeNe power of $P_{\mathrm{HeNe}} \geqslant 100 \mu \mathrm{W}$ and above, there is an increase of $P_{\text {down }}$ until the collapse of the hysteresis loop. Similarly to the temperature experiment, the transmitted intensity in the upper branch of the hysteresis loop decreases steadily until the bistability collapses. The qualitative agreement between both series of experiments strongly suggests that an increase in temperature leads indeed to a larger incoherent population in the reservoir 

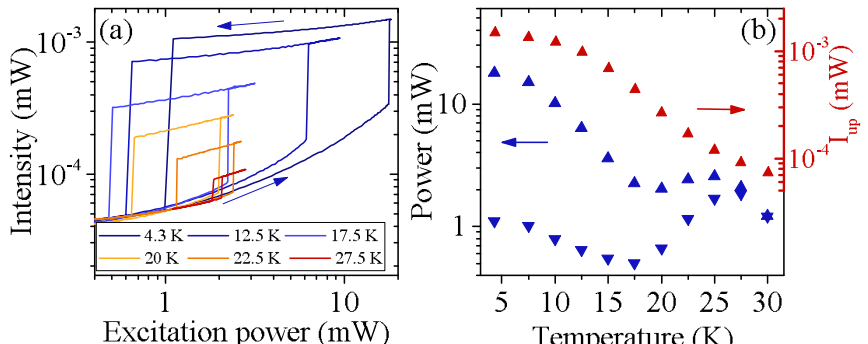

Excitation power $(\mathrm{mW})$
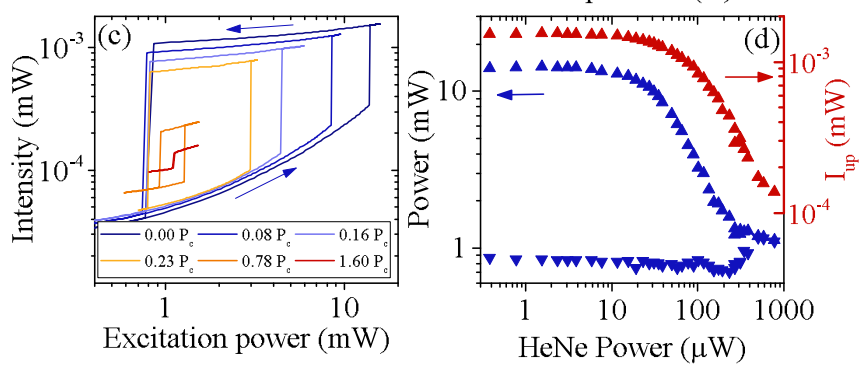

FIG. 3. Polariton bistability for $\Delta=500 \mu \mathrm{eV}$. (a) Hysteresis curves of the transmitted intensity for increasing temperature. The blue arrows indicate the path when cycling the input power. (b) Excitation power at the upward and downward thresholds (blue) and transmitted intensity above the upward threshold (red) for a given temperature. The up (down) triangles correspond to the upward (downward) thresholds. (c, d) The same as in (a) and (b) but for increasing nonresonant laser power. $P_{c}=500 \mu \mathrm{W}$ represents the nonresonant power needed for the hysteresis to collapse.

which interacts with the driven polariton population. Finally, the slight increase of the intensity of the lower branch with increasing HeNe power for resonant excitation below $1 \mathrm{~mW}$ seen in Fig. 2(c) corresponds to unfiltered luminescence from the sample and most likely originates from the thick GaAs layers of the DBR mirrors excited by the HeNe laser.

Although the leveling off of $P_{\text {up }}$ is not pronounced in the temperature experiment, increasing the laser detuning changes this behavior considerably. Results when repeating the same experiments with a laser detuning of $\Delta=500 \mu \mathrm{eV}$ are presented in Fig. 3. For the temperature measurements [Figs. 3(a) and 3(b)], both the leveling off of $P_{\text {up }}$ and the increase of $P_{\text {down }}$ are accentuated and occur for temperatures above $20 \mathrm{~K}$. For the case of nonresonant pumping [Figs. 3(c) and 3(d)], the increase of laser detuning causes only the upward threshold evolution to be shifted to higher power compared to $\Delta=300 \mu \mathrm{eV}$. Because of the larger laser detuning, a higher energy blueshift is needed, and thus a larger reservoir density in order to drive the collapse of the hysteresis loop. For the nonresonant pumping, this only shifts the whole behavior to higher pump power. However, for the case of temperature, in order to reach a higher reservoir density, the temperature must be increased further, leading to possible changes in the reservoir dynamics and scattering coefficients. This is why Figs. 2(a) and 2(b), and Figs. 3(a) and 3(b) differ for temperatures above $20 \mathrm{~K}$.

\section{THEORETICAL MODELING}

We use a simplified model compared to the one presented in Ref. [20] in order to simulate the evolution of the bistability as a function of the reservoir density. We work with the excitonic Bloch equation (EBE) formalism, since it is straightforward to include dephasing [30,41]. Other models based on the master equation for the density matrix of the lower polariton have been proposed to study the effect of temperature on the bistability $[42,43]$. However, these models did not account for the presence of an incoherent reservoir and predicted a symmetrical collapse of both thresholds for temperatures up to $100 \mathrm{~K}$, in contrast to what has been observed here. Polariton bistability under incoherent pumping was theoretically investigated in Ref. [44], but no RID was included in the model. It is worth noting that the dephasing can be implemented by assuming different linewidths for the lower and upper polariton branch when both are coherently excited [29].

Contrary to the Gross-Pitaevskii equations (GPE), where a dephasing is included only as a loss of polariton, in the EBE, a polariton or exciton dephased from the driving laser can still contribute to the blueshift. Both formalisms are linked, as we can recover the GPE in the exciton-photon basis in the coherent limit of EBE [30]. Working in the exciton-photon basis is advantageous since the interaction strengths and couplings are automatically renormalized as a function of pump, reservoir density, and temperature. However, the polariton linewidths can be overestimated because we include exciton and photon linewidth instead of the polariton linewidths directly. This is equivalent to assuming exciton-phonon scattering instead of polariton-phonon scattering. Nonetheless, it is a good tool to qualitatively analyze the system. It is understood that the linewidths in the model are effective and include other effects like experimental noise or other fluctuations.

The model considers the polariton system in the excitonphoton basis at the mean-field level and assumes specific equations of motion for the coherent exciton polarization $P$ and the exciton population $N=|P|^{2}+N_{\text {incoh }}$ ( $N$ does not include the excitons in the reservoir). The EBEs allow us to study decoherence by implementing different loss rates for both coherent and incoherent parts [30]. Following the notation of Ref. [30], the EBEs with additional reservoir terms read

$$
\begin{aligned}
i \hbar \dot{N} & =-i \Gamma_{x} N-2 i \Omega_{P}^{N} \operatorname{Im}\left(P E^{*}\right), \\
i \hbar \dot{P} & =\left(\epsilon_{x}-i \gamma_{x}^{N}+g_{0} N+g_{R} N_{R}\right) P+\Omega_{P}^{N} E, \\
i \hbar \dot{E} & =\left(\epsilon_{c}-i \gamma_{c}\right) E+\Omega_{E}^{N} P+f_{\mathrm{ext}},
\end{aligned}
$$

where the density-dependent parameters are defined as

$$
\begin{gathered}
\Omega_{P}^{N}=\Omega-2 g_{p a e} N-2 g_{p a e}^{R} N_{R}, \\
\Omega_{E}^{N}=\Omega-g_{p a e} N-g_{p a e}^{R} N_{R}, \\
\gamma_{x}^{N}=\Gamma_{x} / 2+g^{\prime} N+g_{R}^{\prime} N_{R}+\gamma^{*} .
\end{gathered}
$$

The term $N_{R}$ is the reservoir population, either created by the HeNe laser (relaxation of hot carriers to form excitons in the reservoir) or through phonon scattering (see below), $E$ is the photon mean field, $f_{\text {ext }}=\sqrt{I_{0}} e^{-i E_{l} t / \hbar}$ is the resonant laser of intensity $I_{0}$ and energy $E_{l}$, and $\epsilon_{x}$ and $\epsilon_{c}$ are the exciton and photon energy. The exciton-exciton interaction strength is noted $g_{0}$, and $g_{R}$ represents the interaction of the coherent exciton with the reservoir. The effect of excitation- 
induced dephasing (EID) is added phenomenologically as in Ref. [30]. We assume that the exciton interaction strength has both a real and an imaginary part, $g=g_{0}-i g^{\prime}$, which leads to the density-dependent exciton polarization linewidth given in Eq. (4), where $\gamma^{*}$ is a pure dephasing mechanism. Finally, the effect of phase-space filling is included through the parameters $g_{\text {pae }}$ and $g_{\text {pae }}^{R}$ that describe the reduction of the coupling strength at high densities. We recover GPE in the coherent limit $\left(N=|P|^{2}\right)$ when all reservoir terms $N_{R}$ are zero as well as $g^{\prime}, g_{\text {pae }}$, and $\gamma^{*}$. The reservoir terms in Eqs. (1) can be derived by considering the reservoir as an effective exciton mode following a similar approach as in Ref. [35] and detailed in the Appendix.

As stated previously, $P_{\text {up }}$ is mainly affected by the polaritonpolariton interaction strength. This implies that $P_{\text {up }}$ decreases from its initial value when the population in the reservoir starts to interact with the resonantly excited polariton. Accordingly, a negative value of the second derivative of the dependence of $P_{\text {up }}$ with the HeNe power indicates a change in the reservoirexciton interaction. Therefore the leveling off of $P_{\text {up }}$ measured is an indicator of the saturation of the reservoir-induced energy blueshift. This effect is not a consequence of the saturation of the Rabi coupling strength $\Omega$ by phase-space filling terms $g_{\text {pae }}$. Indeed, the saturation of the oscillator strength would decrease the Rabi coupling, providing an extra blueshift to the lower polariton mode, hence accelerating the whole process. Other mechanisms that transfer the populations between $N$ and $N_{R}$ would reduce the blueshift induced by one population while increasing the contribution of the other one. The same behavior occurs if we include bosonic stimulation $\left(N_{R}\right.$-dependent gain for the exciton polarization) to deplete the reservoir. This would also lead to an increase of $|P|^{2}$ with $N_{R}$ while the opposite is seen experimentally. Furthermore, increasing the gain would keep the polariton mode locked to the laser for a longer time, resulting in a decrease of $P_{\text {down }}$ with an increase of $\mathrm{HeNe}$ power.

Therefore the leveling off of $P_{\text {up }}$ is either caused by a saturation of the reservoir density with increasing $\mathrm{HeNe}$ power or by a decrease of the interaction strength with density. Photoluminescence measurement while exciting the sample with the HeNe laser showed a linear dependence of the integrated intensity of both $0 \mathrm{D}$ and $2 \mathrm{D}$ polaritons, over the complete range of power available, suggesting that the reservoir population should have a linear dependence with the excitation power. Therefore the variation of reservoir-exciton interaction strength with density is more likely. This could occur, for example, in the case of large momentum transfer between interacting excitons [45], i.e., redistribution of the reservoir population toward a large $k$ vector with increasing excitation density. Since our simplified model does not include a detailed calculation of exciton interactions, we parametrize this decrease of reservoir interaction strength by a sigmoid function that converges asymptotically towards zero: $g_{R} \rightarrow$ $g_{R}\left(N_{R}\right)=2 g_{R} /\left(1+e^{\alpha N_{R}}\right)$.

To fit our data, we simulate a series of bistability curves for increasing $N_{R}$ and minimize the error for $P_{\text {up }}, P_{\text {down }}$, and $I_{\text {up }}$ simultaneously by adjusting the parameters. The exciton and photon energy, the Rabi coupling, and the laser energy are all fixed and are extracted from the experiment, whereas the other parameters are fitted. Each run of experiments can converge
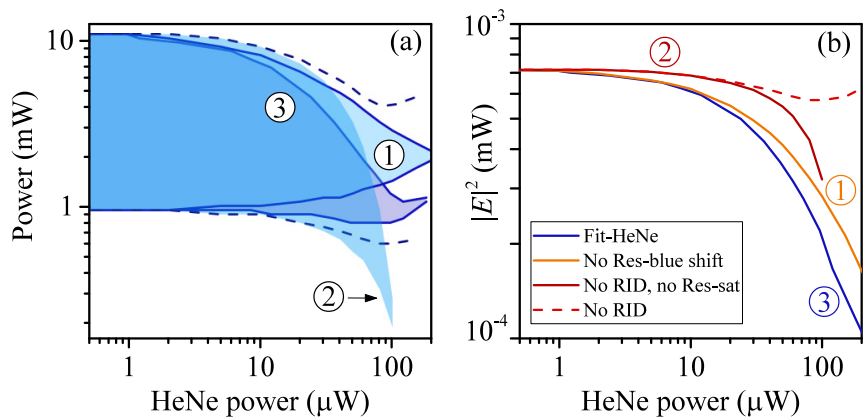

FIG. 4. (a) Simulated upward and downward threshold power for different reservoir parameters. Case 1 is a simulation when $g_{R}$ is set to zero. Case 2 includes $g_{R}$ without the asymptotic decrease with density and with $g_{R}^{\prime}=0$. The dashed line represents the same case with the asymptotic decrease, and case 3 is the fit shown in Fig. 2(d) where all effects are included. All other parameters are kept constant in all cases. (b) Simulated intensity above the upward threshold for the same cases as in (a).

to slightly different sets of parameters, mainly because of intrinsic noise fluctuation between experiments as well as fluctuations of the excitation density. We set $g_{p a e}=g_{p a e}^{R}=$ 0 , for simplicity, since the magnitudes of those terms are always smaller than the corresponding energy blueshift. The parameter $\alpha$ is used to fit the leveling off of $P_{\text {up }}$, and the values of $N_{R}$ and $I_{0}$ are scaled proportionally in order to fit the excitation densities. For the HeNe experiment at $\Delta=$ $300 \mu \mathrm{eV}$, the parameters obtained are $\gamma_{c}=\Gamma_{x}=30 \mu \mathrm{eV}$, $g^{\prime}=0.09 g_{0}, g_{R}=0.4 g_{0}$, and $g_{R}^{\prime}=0.1 g_{0}$. For the temperature experiment at the same $\Delta$, we included the exciton redshift and assumed as a first approximation that the population of the reservoir is proportional to the temperature: $N_{R} \propto a T$. The HeNe experiment has shown that we must include a dephasing term of the type $\gamma_{R I D}=g^{\prime} N_{R}$. At the same time, the effect of temperature is usually considered as a pure dephasing term: a linewidth proportional to temperature $\gamma^{*}=\alpha_{T} T$ [neglecting the LO-phonon term $\left.\gamma_{L O}=b\left(\exp \left[\hbar \omega_{L O} / k T\right]-1\right)^{-1}\right][46]$. We have seen that the effect of temperature is linked to a reservoir population, and hence it is appropriate to equate both equations, which gives $N_{R} \propto a T$. We set $\alpha=0$ since the leveling off is not clearly observed. Under these conditions, the model converged to a similar set of parameters: $\gamma_{c}=$ $30 \mu \mathrm{eV}, \Gamma_{x}=0.5 \mu \mathrm{eV}$ [47], $g^{\prime}=0.024 g_{0}, g_{R}=0.25 g_{0}$, and $g_{R}^{\prime}=0.14 g_{0}$. However, our model did not converge for the temperature data at $\Delta=500 \mu \mathrm{eV}$ with $\alpha \neq 0$, indicating that $N_{R}$ is no longer proportional to $T$ for higher temperatures. The distribution of $N_{R}$ in $k$ space might also evolve with temperature, changing the effective value of $g_{R}$.

Using our best fit parameters, we can study the effect of each $N_{R}$ term on the evolution of the bistability. We focus on the simulations of Fig. 2(d) since the HeNe and temperature experiments lead to the same trend. Figure 4(a) shows the evolution of the thresholds for three specific sets of parameters when $N_{R}$ is increased, case 3 being the fit presented in Fig. 2(d). For case 1 , we set the reservoir-induced blueshift to zero $\left(g_{R}=0\right)$, while all other parameters are fixed. That way, the only contribution from the reservoir is the RID. As can be seen, the increase of RID causes a slow decrease of the upward threshold but, importantly, an increase of $P_{\text {down }}$. 

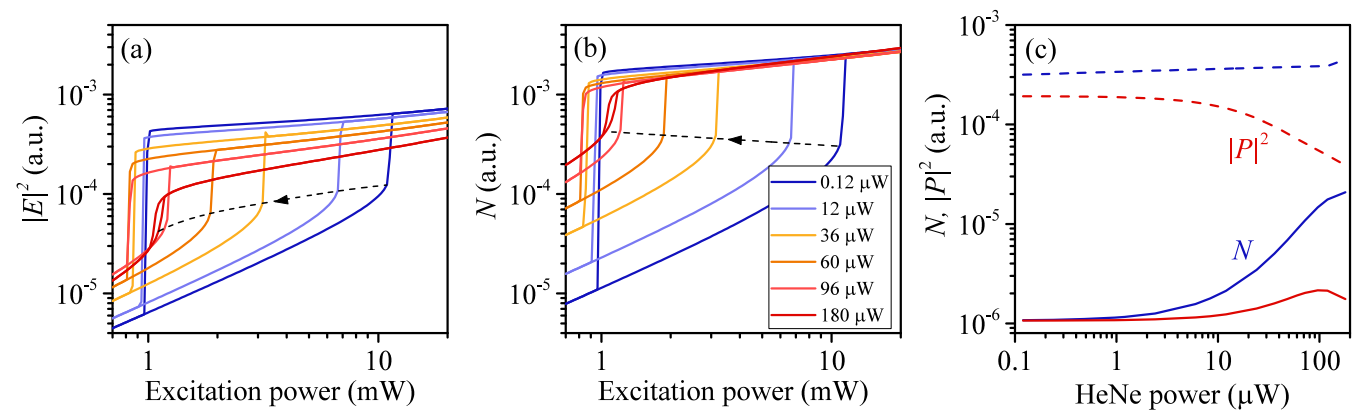

FIG. 5. (a) Simulated photon intensity $|E|^{2}$ as a function of excitation power for different values of incoherent pumping intensity (HeNe power). (b) Simulated exciton population $N=|P|^{2}+N_{\text {incoh }}$ for the same conditions as in (a). The dashed lines indicate the evolution of the coherent (a) and total (b) population before the upward threshold. (c) $N$ and $|P|^{2}$ as a function of HeNe power. The full lines correspond to the population for the initial resonant laser power, whereas the dashed lines correspond to the values at the onset of the upward threshold.

For case 2, we study the opposite scenario as in case 1 $\left(g_{R} \neq g_{R}^{\prime}=0\right)$ while turning off the asymptotic decrease. For low values of $N_{R}, P_{\text {up }}$ is essentially given by $g_{0} N$ and it decreases when $g_{R} N_{R}$ becomes large enough. The same is true for $P_{\text {down }}$; both threshold powers decrease rapidly with $N_{R}$, leading to a quicker collapse of the bistability. Since the term $g_{R} N_{R}$ pushes the polariton mode closer to the resonant laser, both thresholds occur at lower excitation power. Turning on the asymptotic decrease slows down the effect, as shown with the dashed lines. Comparing cases 1 and 2 shows that the measured steady value of $P_{\text {down }}$ is an indicator of the competition between the reservoir-induced blueshift and the RID. Close to the bistability collapse, the increase of $P_{\text {down }}$ shows that the effect of RID is dominant over the energy blueshift induced by the reservoir population.

The RID also affects the intensity in the upper branch of the hysteresis loop [shown in Fig. 4(b)]. For example, if we compare the intensity obtained in cases 2 and 3 (red and blue curve), we see that for a given value of $P_{\text {up }}$, the transmitted intensity is always higher for case 2. The RID must be present in order to obtain the same combination of threshold power and intensity that was measured.

Having access to both the coherent and incoherent populations in the simulation allows us to study the system's coherence. Figures 5(a) and 5(b) show the calculated hysteresis loop of the coherent photon intensity $|E|^{2}$ and of the exciton population $N$ for different reservoir densities (HeNe power). As $N_{R}$ increases, the coherent population at the onset of the upward threshold decreases, as well as $P_{\text {up }}$. Conversely, $N$ slightly increases at the onset, as shown by the dashed line in Fig. 5(b), and it remains relatively constant above the upward threshold. Since the RID broadens the polariton mode, it increases the overlap between the resonant laser and the polariton mode and contributes to the transfer of the polariton population from coherent to incoherent [see Fig. 5(c) and discussion below]. Therefore, the transfer to the incoherent population is enhanced by the interaction of the coherent population with that of the exciton reservoir. It also allows the system to reach the upward threshold for a lesser resonant excitation power.

Finally, the effect of RID is summarized in Fig. 5(c), where we show the evolution of $N$ and $|P|^{2}$ for a resonant laser power of $0.1 \mathrm{~mW}$ (full lines) and at the onset of the upward threshold (dashed lines). The difference between the blue and red curves gives the incoherent exciton population $N_{\text {incoh }}=$ $N-|P|^{2}$, excluding that of the reservoir $N_{R}$. We see that for low resonant power and reservoir density, the system is fully coherent, $N=|P|^{2}$. As the reservoir population increases, the initial incoherent population increases compared to the coherent one, meaning that the resonant laser contributes more efficiently to the incoherent part. This imbalance is maintained at the onset of the upward threshold. For low reservoir density, $N>|P|^{2}$ since $g^{\prime} \neq 0$. As the RID is increased, the onset density increases slightly while the density of the coherent population decreases, which is reflected by the decrease of transmitted laser intensity.

\section{CONCLUSIONS}

We have demonstrated the drastic effect of an incoherent reservoir on a coherent polariton system. Comparing experiments with simulations evidences that a reservoir population can be generated at low temperature, even if the excitation laser is tuned below the reservoir energy. This reservoir either originates from a population transfer of polaritons by means of an acoustic phonon absorption, as suggested in [20], or from exciton-polariton scattering.

We have shown that a loss of coherent population $|P|^{2}$ does not imply a loss of population $N$ of the full system. This explains the decrease of the upward threshold power when the reservoir population is increased, since the energy blueshift is mostly defined by the total exciton population $N$. We also showed that the transmitted intensity is an indicator of the coherent polariton population, whereas the blueshift is an indicator of the total exciton population. When the coherent population decreases, the optical response of the system is dominated by the incoherent population. This implies that evaluating the polariton density using the emission intensity will always underestimate the total polariton density present in the system. Since the latter determines the amplitude of the blueshift and the saturation of the oscillator strength, it should be considered carefully, especially when trying to differentiate between polariton condensate and photon lasing thresholds.

Finally, we have shown that the downward threshold power is an indicator for the RID. Its value increases when the RID is more important to the system than the reservoir-induced 
blueshift. This effect will need to be considered in schemes using polaritons for a high-fidelity quantum light source and quantum information processing. Of course, due to the small binding energy of excitons in InGaAs and the small Rabi energy of our system, the effects described here occur at amazingly low temperatures. Similar effects will also show up, possibly at higher temperatures, in other polariton systems with a larger Rabi splitting.

\section{ACKNOWLEDGMENTS}

The present work is supported by the Swiss National Science Foundation under Project No. 135003 and the European Research Council under project Polaritonics, Contract No. 291120. The Polatom Network is also acknowledged.

\section{APPENDIX: DERIVATION OF THE MULTIMODE EBE EQUATIONS}

Equations (1) can be obtained from the multimode EBE equations, for which we give a brief derivation here. We start with an effective multimode Hamiltonian for the excitonphoton system, which is obtained by performing the Usui transform of the electron-hole-photon Hamiltonian [41], and read:

$$
\begin{gathered}
H=H_{0}+H_{l m}+H_{c}+H_{\text {sat }}, \\
H_{0}=\sum_{i} E_{x i} x_{i}^{\dagger} x_{i}+\sum_{i} E_{c i} c_{i}^{\dagger} c_{i}, \\
H_{l m}=\frac{1}{2} \sum_{i} \Omega_{i}\left(x_{i}^{\dagger} c_{i}+x_{i} c_{i}^{\dagger}\right), \\
H_{c}=\sum_{i j} g_{i j} x_{i}^{\dagger} x_{j}^{\dagger} x_{i} x_{j}, \\
H_{\mathrm{sat}}=-\sum_{i j} g_{p i j}\left(x_{i}^{\dagger} x_{j}^{\dagger} x_{j} c_{i}+x_{i}^{\dagger} x_{i} x_{j} c_{j}^{\dagger}\right),
\end{gathered}
$$

where $H_{0}$ is the single-particle energy, $H_{l m}$ is the light-matter part, $H_{c}$ is the exciton-exciton interaction, and $H_{\text {sat }}$ is the effect of phase-space filling on the exciton-photon coupling. $E_{x i}$ $\left(E_{c i}\right)$ is the exciton (photon) energy in the $i$ th mode, $\Omega_{i}$ is the Rabi splitting between the two, $g_{i j}$ is the exciton-exciton cross-interaction strength, and $g_{p i j}$ is the cross-phase-space filling parameter. This effective Hamiltonian can represent any case of multimode coupling between excitons and photons. For instance, if $i, j=1,2$, this gives the spinor interactions with $g_{11}=g_{22}=\alpha_{1}$ and $g_{12}=g_{21}=\alpha_{2}$. Of course, extra care should be taken to assure momentum conservation between states $i, j$, as well as considering the dependence on the momentum exchange of the exciton-exciton interaction [45]. None of these effects are explicitly considered in this effective Hamiltonian. The mean-field equations are obtained using
Heisenberg's equation of motion for the exciton, photon, and exciton number operator for a given mode $i$. We then define the mean fields as

$$
\begin{gathered}
N_{i}=\left\langle x_{i}^{\dagger} x_{i}\right\rangle, \\
P_{i}=\left\langle x_{i}\right\rangle, \\
E_{i}=\left\langle c_{i}\right\rangle,
\end{gathered}
$$

and use the following approximations in order to conserve the number of particles within each mode: $\left\langle\hat{x}_{i}^{\dagger} \hat{x}_{i} \hat{x}_{j}\right\rangle \cong\left\langle\hat{x}_{i}^{\dagger} \hat{x}_{i}\right\rangle\left\langle\hat{x}_{j}\right\rangle$ and $\left\langle\hat{x}_{i}^{\dagger} \hat{x}_{i}^{\dagger}\right\rangle=\left\langle\hat{x}_{j} \hat{x}_{j}\right\rangle=0 \forall(i, j)[30,48,49]$. Applying these mean-field conditions and adding the mode linewidths gives the multimode EBE:

$$
\begin{aligned}
i \hbar \dot{N}_{i}= & -i \Gamma_{x i}-2 \operatorname{Im}\left(P_{i} E_{i}^{*}\right)\left(\frac{\Omega_{i}}{2}-\sum_{j} g_{p i j} N_{j}\right), \\
i \hbar \dot{P}_{i}= & \left(E_{x i}-i \gamma_{x i}+\sum_{j} g_{i j} N_{j}\right) P_{i} \\
& +\left(\frac{\Omega_{i}}{2}-\sum_{j} 2^{\alpha} g_{p i j} N_{j}\right) E_{i}, \\
i \hbar \dot{E}_{i}= & E_{c i}-i \gamma_{c i}+\left(\frac{\Omega_{i}}{2}-\sum_{j} g_{p i j} N_{j}\right) P_{i},
\end{aligned}
$$

where we have used $E_{i}^{*} P_{i}-P_{i}^{*} E_{i}=2 i \operatorname{Im}\left(P_{i} E_{i}^{*}\right)$ and a factor 2 is included in the term $g_{i j}$. The term $\alpha=1$ if $i=j$ and zero otherwise. If we include a real and imaginary part to the exciton-exciton interactions, we get the following $i$ th exciton linewidth which includes EID from all modes and pure dephasing $\gamma^{*}$ :

$$
\gamma_{x i}=\Gamma_{x i} / 2+\sum_{j} g_{i j}^{\prime} N_{j}+\gamma^{*} .
$$

The coherent limit of Eqs. (A9) $\left(\left|P_{i}\right|^{2}=N_{i}\right)$ occurs for $g_{i j}^{\prime}=g_{p i j}=\gamma^{*}=0$, which gives the multimode GPE in the exciton-photon basis, identical to what has been used for studying the spatial multistability in a large mesa [35]. For $i, j=1,2$, these equations are identical to the spinor GPE (without the biexciton equation) [32]. It is easy to see that we recover EBE with the reservoir [Eqs. (1)] if the summation is over two modes, one exciton in strong coupling, the other being an effective reservoir with no coupling to light (exciton states lying above the light cone), and assuming steady state for the $N_{R}$ equation. From this perspective, the reservoir density-dependent interaction strength $g_{R}\left(N_{R}\right)$ is well justified within this approximation.
[1] I. Carusotto and C. Ciuti, Rev. Mod. Phys. 85, 299 (2013).

[2] T. C. H. Liew and V. Savona, Phys. Rev. Lett. 104, 183601 (2010).

[3] O. Kyriienko and T. C. H. Liew, Phys. Rev. A 90, 063805 (2014).
[4] C. Ciuti, Phys. Rev. B 69, 245304 (2004).

[5] S. Portolan, O. Di Stefano, S. Savasta, and V. Savona, Europhys. Lett. 88, 20003 (2009).

[6] T. C. H. Liew and V. Savona, Phys. Rev. A 84, 032301 (2011). 
[7] T. C. H. Liew and V. Savona, New J. Phys. 15, 025015 (2013).

[8] D. Pagel, H. Fehske, J. Sperling, and W. Vogel, Phys. Rev. A 88, 042310 (2013).

[9] T. Byrnes, K. Wen, and Y. Yamamoto, Phys. Rev. A 85, 040306(R) (2012).

[10] S. S. Demirchyan, I. Y. Chestnov, A. P. Alodjants, M. M. Glazov, and A. V. Kavokin, Phys. Rev. Lett. 112, 196403 (2014).

[11] D. D. Solnyshkov, O. Bleu, and G. Malpuech, Superlattices Microstruct. 83, 466 (2015).

[12] O. Kyriienko and T. C. H. Liew, Phys. Rev. B 93, 035301 (2016).

[13] F. Tassone, C. Piermarocchi, V. Savona, A. Quattropani, and P. Schwendimann, Phys. Rev. B 53, R7642(R) (1996).

[14] V. Savona and C. Piermarocchi, Phys. Status Solidi A 164, 45 (1997).

[15] X. Marie, P. Renucci, S. Dubourg, T. Amand, P. Le Jeune, J. Barrau, J. Bloch, and R. Planel, Phys. Rev. B 59, R2494(R) (1999).

[16] P. Borri, J. R. Jensen, W. Langbein, and J. M. Hvam, Phys. Rev. B 61, R13377(R) (2000).

[17] S. Savasta, O. Di Stefano, V. Savona, and W. Langbein, Phys. Rev. Lett. 94, 246401 (2005).

[18] C. Diederichs, J. Tignon, G. Dasbach, C. Ciuti, A. Lemaître, J. Bloch, P. Roussignol, and C. Delalande, Nature (London) 440, 904 (2006).

[19] M. Romanelli, C. Leyder, J. P. Karr, E. Giacobino, and A. Bramati, Phys. Rev. Lett. 98, 106401 (2007).

[20] D. V. Vishnevsky, D. D. Solnyshkov, N. A. Gippius, and G. Malpuech, Phys. Rev. B 85, 155328 (2012).

[21] T. Paraïso, D. Sarchi, G. Nardin, R. Cerna, Y. Leger, B. Pietka, M. Richard, O. El Daï, F. Morier-Genoud, V. Savona et al., Phys. Rev. B 79, 045319 (2009).

[22] G. Grosso, S. Trebaol, M. Wouters, F. Morier-Genoud, M. T. Portella-Oberli, and B. Deveaud, Phys. Rev. B 90, 045307 (2014).

[23] M. Wouters, I. Carusotto, and C. Ciuti, Phys. Rev. B 77, 115340 (2008).

[24] E. Wertz, L. Ferrier, D. D. Solnyshkov, R. Johne, D. Sanvitto, A. Lemaître, I. Sagnes, R. Grousson, A. V. Kavokin, P. Senellart et al., Nat. Phys. 6, 860 (2010).

[25] L. Ferrier, E. Wertz, R. Johne, D. D. Solnyshkov, P. Senellart, I. Sagnes, A. Lemaître, G. Malpuech, and J. Bloch, Phys. Rev. Lett. 106, 126401 (2011).

[26] G. Tosi, G. Christmann, N. G. Berloff, P. Tsotsis, T. Gao, Z. Hatzopoulos, P. G. Savvidis, and J. J. Baumberg, Nat. Phys. 8, 190 (2012)

[27] A. P. D. Love, D. N. Krizhanovskii, D. M. Whittaker, R. Bouchekioua, D. Sanvitto, S. A. Rizeiqi, R. Bradley, M. S. Skolnick, P. R. Eastham, R. André et al., Phys. Rev. Lett. 101, 067404 (2008).

[28] D. N. Krizhanovskii, K. G. Lagoudakis, M. Wouters, B. Pietka, R. Bradley, K. Guda, D. Whittaker, M. S. Skolnick, B. DeveaudPlédran, M. Richard et al., Phys. Rev. B 80, 045317 (2009).
[29] L. Dominici, D. Colas, S. Donati, J. P. R. Cuartas, M. D. Giorgi, D. Ballarini, G. Guirales, A. Bramati, G. Gigli, F. P. Laussy et al., Phys. Rev. Lett. 113, 226401 (2014).

[30] N. Takemura, M. D. Anderson, S. Trebaol, S. Biswas, D. Y Oberli, M. T. Portella-Oberli, and B. Deveaud, Phys. Rev. B 92, 235305 (2015).

[31] S. S. Gavrilov, A. S. Brichkin, A. A. Demenev, A. A. Dorodnyy, S. I. Novikov, V. D. Kulakovskii, S. G. Tikhodeev, and N. A. Gippius, Phys. Rev. B 85, 075319 (2012).

[32] M. Wouters, T. K. Paraïso, Y. Léger, R. Cerna, F. MorierGenoud, M. T. Portella-Oberli, and B. Deveaud-Plédran, Phys. Rev. B 87, 045303 (2013).

[33] T. K. Paraïso, M. Wouters, Y. Léger, F. Morier-Genoud, and B. Deveaud-Plédran, Nat. Mater. 9, 655 (2010).

[34] R. Cerna, Y. Léger, T. K. Paraïso, M. Wouters, F. MorierGenoud, M. T. Portella-Oberli, and B. Deveaud, Nat. Commun. 4, 2008 (2013).

[35] C. Ouellet-Plamondon, G. Sallen, F. Morier-Genoud, D. Y. Oberli, M. T. Portella-Oberli, and B. Deveaud, Phys. Rev. B 93, 085313 (2016).

[36] H. Abbaspour, G. Sallen, S. Trebaol, F. Morier-Genoud, M. T. Portella-Oberli, and B. Deveaud, Phys. Rev. B 92, 165303 (2015).

[37] H. Abbaspour, S. Trebaol, F. Morier-Genoud, M. T. PortellaOberli, and B. Deveaud, Phys. Rev. Lett. 113, 057401 (2014).

[38] H. Abbaspour, S. Trebaol, F. Morier-Genoud, M. T. PortellaOberli, and B. Deveaud, Phys. Rev. B 91, 155307 (2015).

[39] O. El Daif, A. Baas, T. Guillet, J.-P. Brantut, R. I. Kaitouni, J. L. Staehli, F. Morier-Genoud, and B. Deveaud, Appl. Phys. Lett. 88, 061105 (2006)

[40] A. Baas, J. P. Karr, H. Eleuch, and E. Giacobino, Phys. Rev. A 69, 023809 (2004).

[41] G. Rochat, C. Ciuti, V. Savona, C. Piermarocchi, A. Quattropani, and P. Schwendimann, Phys. Rev. B 61, 13856 (2000).

[42] E. B. Magnusson, I. G. Savenko, and I. A. Shelykh, Phys. Rev. B 84, 195308 (2011).

[43] O. Bozat, I. G. Savenko, and I. A. Shelykh, Phys. Rev. B 86, 035413 (2012).

[44] O. Kyriienko, E. A. Ostrovskaya, O. A. Egorov, I. A. Shelykh, and T. C. H. Liew, Phys. Rev. B 90, 125407 (2014).

[45] C. Ciuti, V. Savona, C. Piermarocchi, A. Quattropani, and P. Schwendimann, Phys. Rev. B 58, 7926 (1998).

[46] P. Borri, W. Langbein, J. M. Hvam, and F. Martelli, Phys. Rev. B 59, 2215 (1999).

[47] The value of $\Gamma_{x}$ is lower in the temperature fit compared to the HeNe fits, because it is the temperature term that fixes the exciton linewidth.

[48] S. Mukamel, Principles of Nonlinear Optical Spectroscopy (Oxford University Press, New York, 1995).

[49] N. Takemura, S. Trebaol, M. D. Anderson, V. Kohnle, Y. Leger, D. Y. Oberli, M. T. Portella-Oberli, and B. Deveaud, Phys. Rev. B 92, 125415 (2015). 